\subsection{5 doi: 10.15789/2220-7619-2018-4-6.25 \\ PECULIARITIES OF THE TUBERCULOSIS, HIV AND HIV-ASSOCIATED TUBERCULOSIS INCIDENCE IN THE FAR EASTERN FEDERAL DISTRICT OF THE RUSSIAN FEDERATION}

T.V. Korita ${ }^{1}$, K.V. Zhmerenetsky ${ }^{2}$, E.A. Bazykina ${ }^{1}$, L.A. Balakhontseva ${ }^{1}$

${ }^{1}$ Khabarovsk Research Institute of Epidemiology and Microbiology of Rospotrebnadzor, Khabarovsk, Russia; ${ }^{2}$ Far Eastern Medical

University of the Helthcare Ministry of the Russia, Khabarovsk, Russia

Objective of the research was to analyze the dynamics of the tuberculosis (TB), HIV and HIV-associated TB incidence in the Far Eastern Federal District (FEFD) of the Russian Federation.

The data of the official statistical forms No. 8, No. 33, No. 61 was used. Modern statistical methods were applied.

The TB incidence declined by $43.2 \%$ (from 85.1 to 48.3\%ooo) during 2008-2017 in the Russian Federation when prevalence declined by $42.2 \%$ (from 190.7 to $109.8 \%$ ooo). The most pressing tuberculosis epidemiological situation persisted in the FEFD. During a decline of TB-incidence in the FEFD in the years of $2009-2017$ by $32.9 \%$ the regional index was still higher compared to the Russian-wide rate by $1.8-1.8$ times. HIV-infection in 2017 reached $36.0 \% 000$ versus $14.0 \%$ ooo registered in 2008 in the FEFD. During the analyzed period HIV-incidence accession rate in the FEFD constituent entities changed repeatedly. In 2017 the HIV prevalence rate in FEFD was lower than the Russian-wide index by 2.2 times. The highest HIV prevalence in the FEFD was registered in the Primorsky, Sakhalin Territories and the Chukotka Autonomous Region (57.3; 50.9 и 50.0\% ooo respectively). The HIV-TB associated incidence in the Russian Federation reached 8.3\%ooo in 2017 versus 4.3\%ooo in 2009. During the analyzed period of time percent of the HIV-positive patients with associated TB increased from 6.4 to $17.2 \%$ in the Russian Federation. In the FEFD the same index raised from 2.0 to $4.8 \%$. This said the Russian-wide index consistently exceeded the FEFD rate by 3.1-4.6 times. The highest accession rates of the present index in the FEFD were registered in the Khabarovsk Territory - 9.9\%, the Amur region 9.4\%, the Republic Sakha (Yakutia) $-6.6 \%$.

The FEFD is a leading territory of TB-incidence for several years. An increase in the number of HIV-positive people as well as HIV-TB co-infected patients was registered in the region. Substantial differences of the analyzed indices in different constituent entities of the FEFD were registered.

\section{GLOBAL WHO POLICIES ON MOLECULAR METHODS FOR TB DIAGNOSIS}

A. Korobitsyn

WHO Global TB Program, Geneva, Switzerland

The World Health Organization's (WHO) global strategy for TB prevention, care and control for 2015-2035 (known as the End TB strategy) calls for the early diagnosis of TB and universal drug susceptibility testing (DST), highlighting the critical role of laboratories for rapidly and accurately detecting TB and drug resistance. This requires ensuring access to WHO-recommended rapid diagnostics and universal access to drug-susceptibility testing (DST) for all patients with signs and symptoms of TB. WHO defines universal access to DST as rapid DST for at least rifampicin among all patients with bacteriologically confirmed TB, and further DST for at least fluoroquinolones among all TB patients with rifampicin resistance. The WHO estimates that 10.4 million persons developed tuberculosis (TB) worldwide in 2016, including 490000 cases of multidrug-resistant TB (MDR-TB) and 110000 cases of rifampicin-resistant TB (RR-TB), needing the same second-line treatment regimen.

The objective of the study was to present WHO policies on molecular methods for TB diagnosis.

Xpert MTB/RIF and/or Ultra are recommended as initial test for diagnosis of all persons with signs and symptoms of TB. Line probe assays are recommended as rapid diagnostic tests for detection of resistance to Isoniazid, Rifampicin, Fluoroquinolones and Amikacin. DNA Sequencing is becoming increasingly important as a reference method for detecting mutations associated with resistance to first and second line anti-TB drugs.

According to WHO policies, molecular methods play critical role in global fight with TB.

\subsection{7} doi: 10.15789/2220-7619-2018-4-6.27

\section{PREVALENCE OF NONTUBERCULOUS} MYCOBACTERIUM spp. STRAINS ISOLATED FROM CLINICAL SPECIMENS AT NORTH ESTONIA MEDICAL CENTRE IN 2001-2017

\section{K. Levina}

North Estonia Medical Centre, Tallinn, Estonia

Mycobacteria are widespread microbes in nature. Mycobacteria that are not causative agents of human and animal tuberculosis are designated as non-tuberculous mycobacteria (NTM). NTM caused infections start to become more frequent in the recent years. This report summarizes data of the NTM isolates reported at North Estonia Medical Centre in the period 2001-2017. In Estonia, physicians are not requested to report infections involving NTM species. Reports of the prevalence of MTBC are available in the Estonian Tuberculosis Registry. In contrast, reporting of NTM suspected to be involved in a disease, does not represent the absolute occurence and distribution of the NTM species and disease. These reports are not verified and any association with clinical data should be interpreted with caution.

The incidence of tuberculosis in Estonia shows a trend of decrease. The aim of the study was to analyse the prevalence of NTM strains on the basis of clinical specimens.

The NTM species were isolated from clinical material that was sent to the mycobacteriology laboratory for diagnostic purposes during 2001-2015. The pathological material was decontaminated by $\mathrm{NaOH}+\mathrm{NALC}$ and cultured on Löwenstein-Jensen and Middlebrook media. The NTM species were identified by the GenoType Mycobacterium CM/AS test (Hain Lifescience GmbH).

In 2001-2017, of 114907 investigated specimens, 12790 (10.9\%) MTBC and 1020 (1.04\%) NTM strains were identified. The leading NTM species isolated were Mycobacterium avium $(41.7 \%)$, M. gordonae $(17.5 \%)$, M. fortuitum $(9.3 \%)$ and $M$. intracellulare (6.8\%). The rarely isolated NTM were represented by M. kansasii, M. xenopi, M. szulgai and M. abscessus. Of the NTM, 72\% were cultured from sputum, $5 \%$ from blood and 23\% from other materials. In total, 85 patients were infected with NTM in 2001-2005, 210 in 2006-2010 and 331 in 2011-2017. Of the NTM infected patients, 35\% were $>65$ years old and $9 \%$ were $<30$ years old. A TB and HIV coinfection was found in $317(8.3 \%)$ and NTM-HIV coinfection was found in $56(10.5 \%)$ patients.

During the last 17 years the prevalence of NTM in patients' material has increased approximately three times. The most prevalent species is M. avium. 